\title{
Prognostic Significance of Pregnancy Zone Protein and Its Correlation with Immune Infiltrates in Hepatocellular Carcinoma
}

This article was published in the following Dove Press journal: Cancer Management and Research

\author{
Lisa $\mathrm{Su}^{1, *}$ \\ Genhao Zhang $\mathbb{D}^{2, *}$ \\ Xiangdong Kong (1D \\ 'Department of Genetic and Prenatal \\ Diagnosis Center, Zhengzhou University \\ First Affiliated Hospital, Zhengzhou, \\ People's Republic of China; ${ }^{2}$ Department \\ of Blood Transfusion, Zhengzhou \\ University First Affiliated Hospital, \\ Zhengzhou, People's Republic of China \\ *These authors contributed equally to \\ this work
}

\begin{abstract}
Aim: Human pregnancy zone protein (PZP) is a pregnancy-related protein which is increased dramatically during pregnancy. However, the expression of PZP and its prognostic value, association with tumor-infiltrating immune cells (TIICs) in microenvironment and potential biological process in HCC were unclear.

Methods: The PZP expression, clinicopathology analysis and its influence on survival were analyzed by GEPIA and HPA. Fifty-nine HCC samples and 30 corresponding noncancerous tissues were collected and retrospectively analyzed to verify the results of bioinformatics analysis. Further, TIMER and CIBERSORT were performed to identify the significantly alerted biological process and affections of PZP expression on the immune system in patients with HCC. Finally, IHC assay of CD4+ T cells and Treg cells was performed to confirm the results of immune infiltrates analysis by TIMER and CIBERSORT.
\end{abstract}

Results: PZP expression was downregulated in HCC tissues and its low level was substantially correlated with poor prognosis in patients with HCC. TIMER analysis showed that PZP expression had a positive correlation with the levels of macrophage and neutrophil. Furthermore, CIBERSORT analysis showed that resting memory CD4 T cells were increased in high PZP expression group, while the results of Tregs were the opposite. Finally, the IHC results of CD4+ $\mathrm{T}$ cells and Treg cells showed that only Tregs were negatively associated with PZP expression.

Conclusion: PZP was identified as a novel prognosis biomarker of HCC and might play a vital role in the regulation and recruitment of TIICs in HCC immune microenvironment.

Keywords: PZP, prognosis, TIICs

\section{Introduction}

Hepatocellular carcinoma (HCC), as the main histological type of primary liver cancer, is one of the most common malignancies and leading cause of cancerrelated deaths in China. ${ }^{1}$ The progression of HCC has a significant correlation with $\mathrm{HBV}$ or HCV infection, alcohol abuse, cirrhosis, nonalcoholic fatty liver disease (NAFLD) and aflatoxin exposure. ${ }^{2}$ In fact, due to lack of biomarkers for early diagnosis and curative treatments, more than half of the patients with HCC have reached the late stage at diagnosis. It is known that most of the patients with HCC received treatment such as hepatectomy, embolization therapy (transarterial embolization or transcatheter arterial chemoembolization), radiofrequency ablation, liver transplantation, and molecule-targeted chemotherapy (multikinase inhibitor), however, their prognosis remains unsatisfactory, and quite a few patients still
Correspondence: Xiangdong Kong

Email kongxdprenatal@I63.com
Cancer Management and Research 2020:12 9883-9891 
unfortunately suffered from tumor recurrence several months or years later. ${ }^{3}$ Hence, new interventions for prognostic biomarkers and therapeutic targets to improve the poor prognosis for patients with HCC remains crucial.

Pregnancy zone protein (PZP) is an estrogen-dependent high molecular weight glycoprotein, the level of which could increase dramatically during pregnancy. The concentration of PZP in womens' blood plasma is detectable a few weeks postconception, then can reach up to $3 \mathrm{mg} /$ $\mathrm{mL}$ in some individuals by 30 weeks of gestation, and finally return to near zero immediately postpartum. ${ }^{4}$ As a broad-spectrum immunosuppressant with antiproteinase activity, knockdown of PZP may fail to prevent rejection of heart allografts and increase susceptibility to viral infection in mice. ${ }^{5}$ Furthermore, PZP may have been shown to play an important role in modulating $\mathrm{T}$ cell activation and IL-2 production. ${ }^{6}$ However, the function of PZP has never been previously reported in tumors, especially in HCC. The present study was designed to investigate the relationship among the expression profile of PZP, clinical pathological characteristics and tumor-infiltrating immune cells (TIICs) in patients with HCC, and to explore the role of PZP in HCC development, which might help us understand the mechanisms underlying HCC.

\section{Materials and Methods}

\section{Bioinformatics Analysis by GEPIA and HPA}

The Gene Expression Profiling Interactive Analysis (GEPIA) $^{7}$ (http://gepia.cancer-pku.cn/), which adopts a standard processing pipeline to analyze the RNA-Seq expression data from GTEx and TCGA, was used to explore the association between PZP expression and clinicopathologic characteristics in samples with or without HCC. Survival curve and pathological stage plot of differential PZP expressions were analyzed by GEPIA to investigate the correlation between PZP expression and the prognosis of patients with HCC. Protein expression of PZP between normal and HCC tissues was analyzed by the Human Protein Atlas database (HPA) (www.proteina tlas.org). Cox regression analysis performed with $\mathrm{R}$ software was used to identify whether PZP was an independent prognostic factor in TCGA database.

\section{Clinical Data}

Fifty-nine HCC samples and 30 corresponding noncancerous tissues (normal tissues) from patients admitted to the
Department of Pathology of the First Hospital of Zhengzhou University from 2013 to 2014 were selected. None of the patients were treated with radiotherapy or chemotherapy before surgery and those with incomplete data were excluded. Patients enrolled were approved by the ethics committee of the First Affiliated Hospital of Zhengzhou University. All pathological data were reviewed and joint diagnoses were made by two senior pathologists. Follow-up data were available for all patients up to January 2018, with a follow-up time ranging from 1 to 60 months. Quantitative real-time PCR (qPCR) and immunohistochemistry staining assay (IHC) were performed to assess the mRNA and protein expression levels of PZP between HCC and normal tissues. The total RNA was extracted using TRIzol reagent from tissues and then reverse-transcribed using a PrimeScript RT Master Mix Synthesis Kit for mRNAs as we reported previously. ${ }^{8}$ The relative expression levels of mRNAs were quantified using Maxima SYBR Green qRT-PCR Master Mix $(2 \times)$ in a Real-Time PCR system. The expression level of $\beta$-ACTIN was used as endogenous controls, and the relative mRNA levels of PZP expression were determined using the delta Cycle threshold (Ct) method $^{9}$ and expressed as average $\pm \mathrm{SD}$. The primer sequences are listed as follows: PZP, F: CACAGGAAACCGTCCTGCTT, R: ACTCAG CAACCACAGACTCAT and $\beta$-ACTIN, F: CGTGGGCCGCCCTAGGCA CCA， R: TTGGCTT AGGGTTCAGGGGGG. Sections of formalin-fixed paraffin-embedded HCC and normal tissues were stained using human anti-PZP antibodies (\#HPA038848, Atlas Antibodies) as primary antibody and HRP-conjugated goat anti-mouse IgG (\#NO. D110073, Sangon Biotech) as secondary antibody. Sections were finally stained with 3,3'diaminobenzidine (DAB, \#NO. A690009, Sangon Biotech). A final score of the sum of the extent of expression score (no positive cells $=0,<10 \%=1,10 \%-50 \%=2$, positive staining of $>50 \%=3$ ) and intensity score (negative $=0$, weak $=1$, moderate $=2$, strong $=3$ ) was used to distinguish between negative $(\leq 4)$ and positive $(>4)$ expression of HM13 in HCC and noncancerous tissues.

\section{Immune Infiltrates Analysis by TIMER and CIBERSORT}

In order to evaluate the potential relationships between PZP expression profiles and TIICs in HCC microenvironment, including CD4 $+\mathrm{T}$ cells, CD8 $+\mathrm{T}$ cells, $\mathrm{B}$ cells, dendritic cells, neutrophils and macrophages, the TIMER (http://cis trome.shinyapps.io/timer/) as a comprehensive resource was 
employed. ${ }^{10}$ Furthermore, to assess the influence of PZP expression on the microenvironment of the immune system in HCC sample, 89 of the 365 samples in TCGA database, which had a $p$-value estimated by CIBERSORT less than 0.05 and were divided into two groups according to the quartile value of PZP expression, were used to gauge the proportions of 22 TIICs via CIBERSORT ${ }^{11}$ (http://cibersort. stanford.edu/). Finally, a violin plot was made from the results above. In order to further confirm the results of immune infiltrates analysis by TIMER and CIBERSORT, IHC assay of CD4+ T cells and Treg cells was performed. What is more, the association between PZP and 15 potentially targetable immune checkpoint genes ${ }^{12}$ including CCL2, CD274, CD276, CD4, CTLA4, CXCR4, IL1A, IL6, LAG3, PDL1, PDL2, TGFB1, OX40, CD137 and CDX40L, was analyzed in TCGA database.

\section{Statistical Analysis}

Statistical analyses were performed with $\mathrm{R}$ software (Version 3.6.2). Categorical data were compared with Pearson chi-squared test or Fisher's exact test whenever appropriate. Kaplan-Meier survival analysis, with the logrank test, was used to assess the effect of PZP expression on the OS and RFS of patients with HCC. Cox proportional hazards regression model was used to analyze the association between PZP expression, along with other clinical factors, and overall survival. A $P$-value $<0.05$ for PZP expression was considered statistically significant.

\section{Results \\ Bioinformatics Analysis by GEPIA Demonstrates That Downregulated PZP Expression Predicts Poor Prognosis in HCC Samples}

The results analyzed by GEPIA showed that PZP expression level in normal liver tissues was significantly higher than that in HCC tissues $(p<0.05$, Figure $1 \mathrm{~A})$, while no correlation was found between PZP expression and different tumor stages ( $p>0.05$, Figure 1B). Survival analysis showed that low PZP expression was substantially correlated with poor OS and RFS in patients with HCC (all $p<0.05$, Figure 1C). Furthermore, differential protein expression of PZP between normal and HCC tissues analyzed by HPA was consistent with the results analyzed by GEPIA (Figure 1D). Univariate and multivariate Cox regression analysis was performed after raw expression data and related clinical pathological information of LIHC downloaded from The Cancer Genome Atlas (TCGA) database (https://cancergenome.nih.gov/), the results of which revealed that PZP expression $(\mathrm{HR}=0.871$,
A

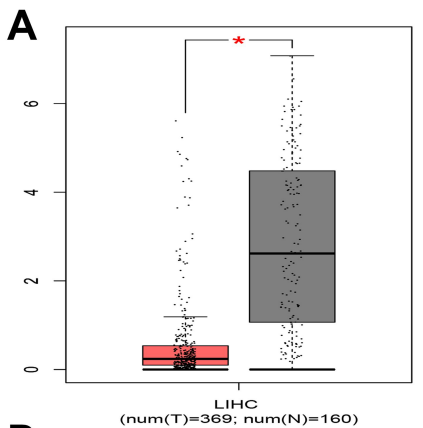

D

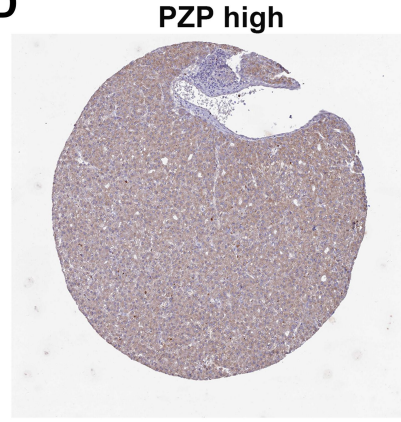

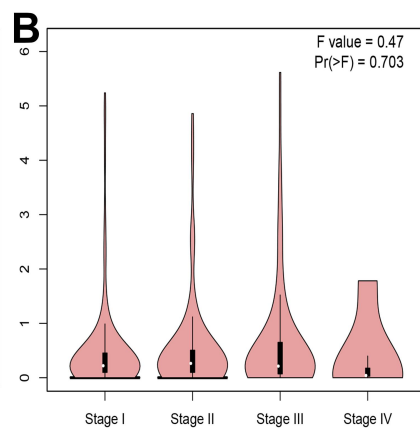

PZP low

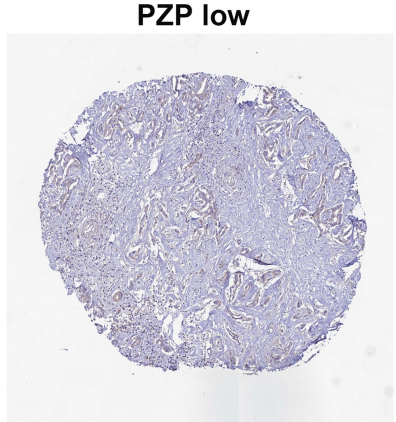

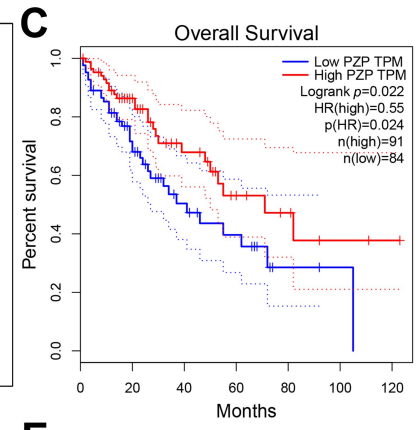

E
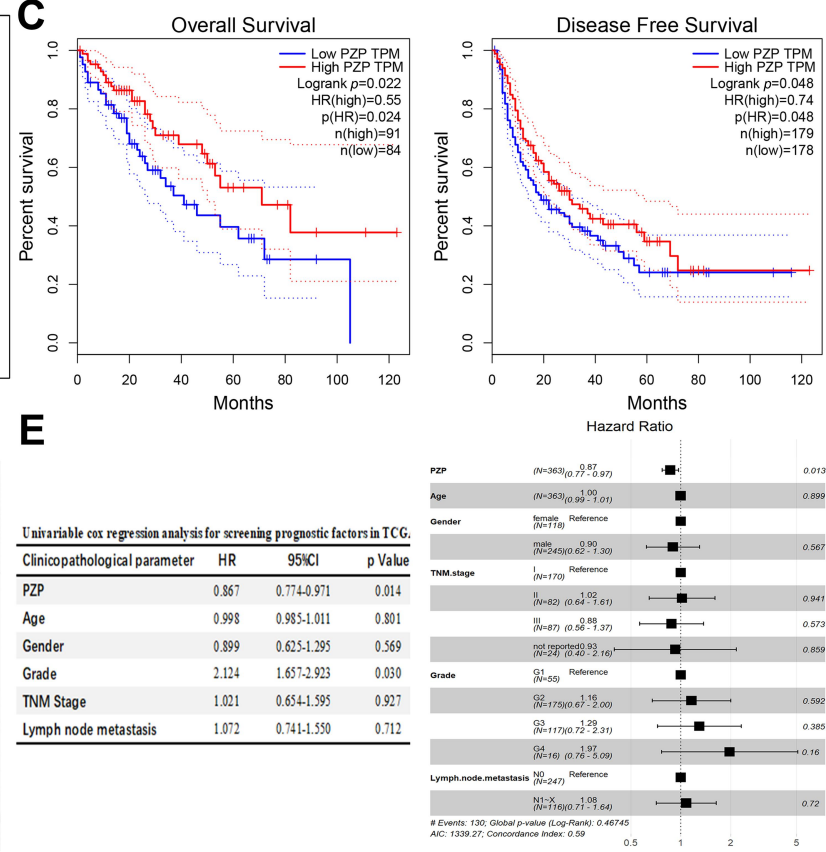

Figure I Bioinformatics analysis by GEPIA and HPA. PZP expression among normal samples, HCC samples (A) and different tumor stages (B). (C) Low PZP expression was substantially correlated with poor OS (C left) and DFS (C right) in patients with HCC. PZP protein expression between normal and HCC tissues analyzed by HPA (D). (E) Univariate (E left) and multivariate (E right) Cox regression analysis. $*_{p}<0.05$. 
95\%CI: $0.776-0.970, p=0.013$ ) was an independent prognostic factor in TCGA database (Figure 1E). In conclusion, PZP expression might play a critical role in the progression of HCC.

\section{PZP Expression is Significantly Decreased in Clinical Tumor Samples and Correlates with Worse Clinical Outcomes in HCC}

In order to verify the results of bioinformatics analysis, 59 HCC samples and 30 corresponding noncancerous tissues were collected. The results of IHC assay (Figure 2A) showed that PZP was expressed in 20 cases of the 59 HCC tissues (33.9\%) and 26 cases of the 30 normal liver tissues $(86.7 \%)$ to a certain degree $(p<0.001$, Figure 2B). The results of qPCR assay showed that the mRNA expression level of PZP was lower in HCC tissues than normal tissues $(p<0.05$, Figure 2C). In summary, PZP expression levels, both mRNA and protein expression, were significantly decreased in HCC tissues, suggesting a positive correlation between HCC and PZP expression. Next, we reanalyzed the data to investigate the role of PZP in the development of HCC. However, there was no difference of PZP expression among different tumor stages (data not shown) and PZP expression was not related to any clinical pathological characteristics including age, gender, lymph node metastasis, TNM stage and WHO grade (all $p>0.05$, Table 1), which was in accord with the results of bioinformatics analysis. Furthermore, patients with lower PZP expression were more likely to be with poor OS no matter according to mRNA expression level or protein expression level (Figure 2D). Subsequently, PZP expression was confirmed as the only independent prognostic factor for patients with $\mathrm{HCC}$ by Cox proportional regression model (PZP negative vs PZP positive: $\mathrm{HR}=2.061,95 \% \mathrm{CI}: 1.003-6.275, p=0.032$, Table 2). From the above, the dysregulated expression of PZP might contribute to the progression of HCC.
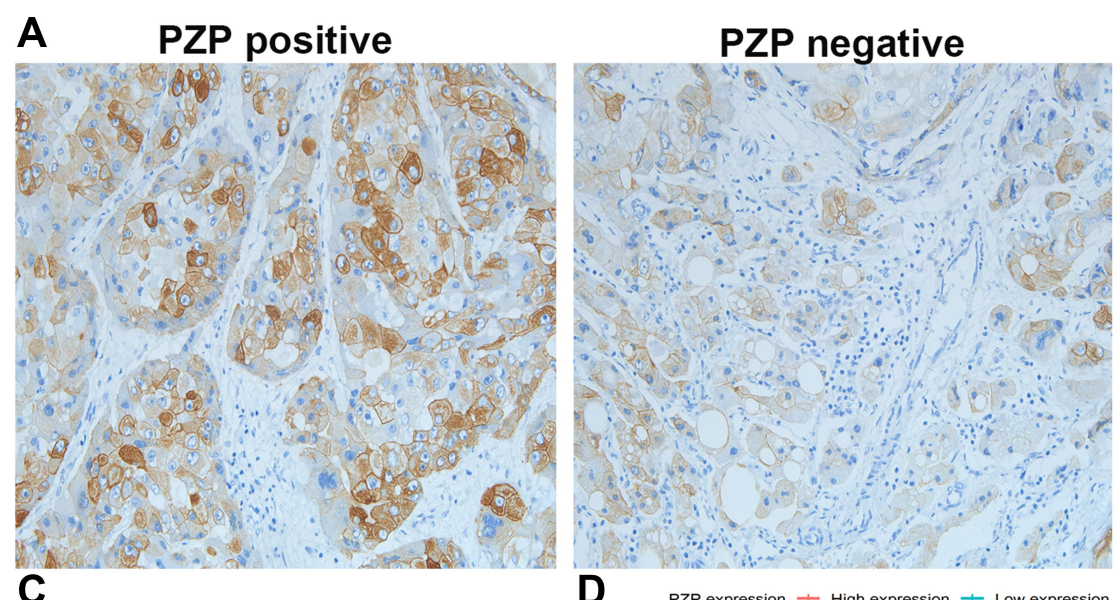

B
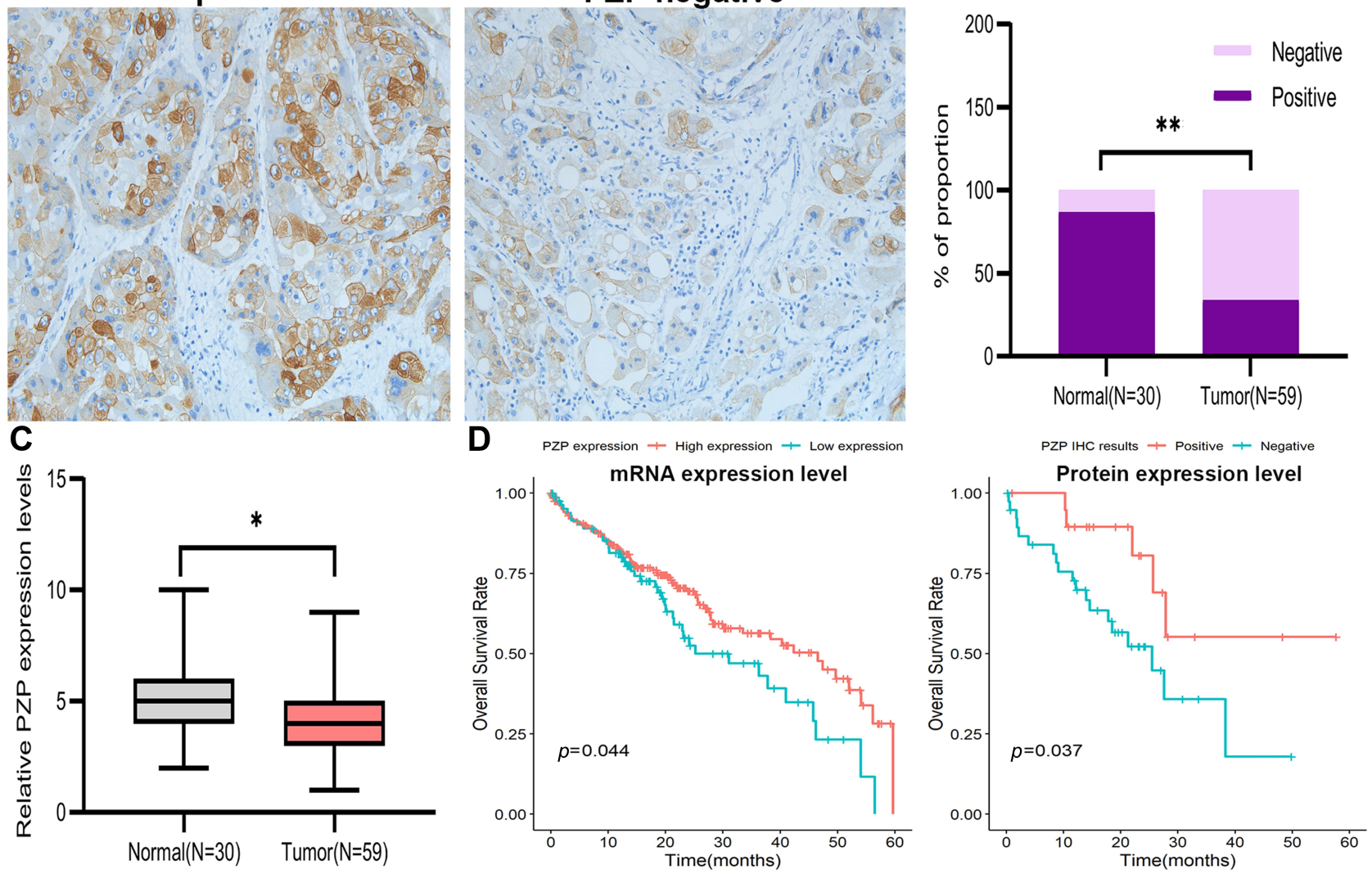

Figure 2 PZP expression in clinical tumor samples and its correlations with clinical outcomes in HCC. (A) PZP protein expression in HCC. EnVision $\times 200$. (B, C) PZP protein and mRNA expression in normal tissues and HCC tissues. (D) OS was compared between patients with or without lower PZP expression according to mRNA expression level ( $D$ left) and protein expression level ( $D$ right). ${ }^{*} p<0.05, * * p<0.00$ I. 
Table I Relationship Between PZP Expression and Clinical Pathological Features

\begin{tabular}{|c|c|c|c|c|c|}
\hline \multirow[t]{2}{*}{ Clinicopathological Parameter } & \multirow[t]{2}{*}{ Case (n) } & \multicolumn{2}{|c|}{ PZP Expression } & \multirow[t]{2}{*}{$\chi^{2}$} & \multirow[t]{2}{*}{$p$-value } \\
\hline & & Positive & Negative & & \\
\hline $\begin{array}{l}\text { Age (years) } \leq 60 \\
>60\end{array}$ & $\begin{array}{l}27 \\
32\end{array}$ & $\begin{array}{l}10 \\
10\end{array}$ & $\begin{array}{l}17 \\
22\end{array}$ & 0.219 & 0.639 \\
\hline $\begin{array}{l}\text { Gender male } \\
\text { Female }\end{array}$ & $\begin{array}{l}45 \\
14\end{array}$ & $\begin{array}{l}17 \\
3\end{array}$ & $\begin{array}{l}28 \\
11\end{array}$ & 1.274 & 0.259 \\
\hline $\begin{array}{l}\text { HBV infection, yes } \\
\text { No }\end{array}$ & & $\begin{array}{l}12 \\
8\end{array}$ & $\begin{array}{l}29 \\
10\end{array}$ & 1.134 & 0.256 \\
\hline $\begin{array}{l}\text { NASH, yes } \\
\text { No }\end{array}$ & $\begin{array}{l}39 \\
20\end{array}$ & $\begin{array}{l}13 \\
4\end{array}$ & $\begin{array}{l}26 \\
16\end{array}$ & 1.146 & 0.284 \\
\hline $\begin{array}{l}\text { Alcohol abuse, yes } \\
\text { No }\end{array}$ & $\begin{array}{l}25 \\
34\end{array}$ & $\begin{array}{l}5 \\
15\end{array}$ & $\begin{array}{l}20 \\
19\end{array}$ & 3.740 & 0.053 \\
\hline $\begin{array}{l}\text { Lymph Node Metastasis } \\
\text { Yes } \\
\text { No }\end{array}$ & $\begin{array}{l}12 \\
47\end{array}$ & $\begin{array}{l}4 \\
16\end{array}$ & $\begin{array}{l}8 \\
31\end{array}$ & 0.002 & 0.963 \\
\hline $\begin{array}{l}\text { Recurrence, yes } \\
\text { No }\end{array}$ & $\begin{array}{l}29 \\
30\end{array}$ & $\begin{array}{l}14 \\
6\end{array}$ & $\begin{array}{l}15 \\
24\end{array}$ & 5.261 & 0.021 \\
\hline $\begin{array}{l}\text { TNM Stage I and II } \\
\text { III\&IV }\end{array}$ & $\begin{array}{l}42 \\
17\end{array}$ & $\begin{array}{l}16 \\
4\end{array}$ & $\begin{array}{l}26 \\
13\end{array}$ & 1.146 & 0.284 \\
\hline $\begin{array}{l}\text { Grade } \mathrm{Gl} \text { and } \mathrm{G} 2 \\
\mathrm{G} 3 \text { and } \mathrm{G} 4\end{array}$ & $\begin{array}{l}35 \\
24\end{array}$ & $\begin{array}{l}11 \\
9\end{array}$ & $\begin{array}{l}24 \\
15\end{array}$ & 0.234 & 0.628 \\
\hline
\end{tabular}

\section{Association Between PZP Expression and TIICs in HCC Microenvironment}

Previous studies have reported that TIICs play an important role in tumor development and prediction of OS rates. Therefore, the results analyzed by TIMER (Figure 3A) showed that PZP expression had a positive correlation with the levels of macrophage ( $p=4.75 \mathrm{e}-05)$ and neutrophil $(p=7.58 \mathrm{e}-06)$, indicating that PZP expression played a potential role in immune infiltration in HCC. Furthermore, differences of HCC immune microenvironment in patients with or without high PZP expression were analyzed by CIBERSORT. The proportions of 22 immune cell subtypes in the two groups were shown in Figure 3B. When compared with low PZP expression group, resting memory CD4 T cells were increased in high PZP expression group, while the results of Tregs were opposite (all $p<0.05)$. In order to further confirm the association between PZP expression and TIICs in HCC microenvironment, IHC assay of CD4+ T cells and Treg cells in HCC tissues was performed. The stained CD4 $+\mathrm{T}$ cells and Treg cells at $200 \times$ magnification in five most representative areas of each HCC tissues were evaluated and finally the mean value was adopted as the count of immune cells per high-power field. ${ }^{13}$ Interestingly, as shown in Figure 3C, only the IHC result of Treg was negatively associated with PZP expression. What is more, after querying the pathological data, we found that Treg cells were significantly increased in recurrent HCC samples while Treg cells had no difference between HCC samples with or without metastasis (Figure 3D). Finally, we investigated the association between PZP and 15 potentially targetable immune checkpoint genes in TCGA database, the results of which showed that high PZP group exhibited higher expression of seven immune checkpoint genes, including CCL2, PDL1, CD4, CTLA4, CXCR4, LAG3 and PDL2, than low PZP group (Figure 3E). All the results above indicated that PZP expression had a significant meaning in regulating tumor immune microenvironment of HCC.

\section{Discussion}

With the characteristics of high malignancy and rapid progress, more than half of patients with HCC were diagnosed at late stage and had a worse clinical outcome. Most 
Table 2 Univariable and Multivariate Cox Regression Analysis for Screening Prognostic Factors

\begin{tabular}{|c|c|c|c|c|}
\hline \multirow[t]{2}{*}{ Clinicopathological Factors } & \multicolumn{2}{|l|}{$\begin{array}{l}\text { Univariable Cox } \\
\text { Regression Analysis }\end{array}$} & \multicolumn{2}{|c|}{ Multivariable Cox Regression Analysis (Forward) } \\
\hline & HR (95\%Cl) & p-value & HR (95\% Cl) & p-value \\
\hline $\begin{array}{l}\text { PZP Expression } \\
\text { Negative vs positive }\end{array}$ & $2.745(1.019-7.393)$ & 0.046 & $2.061(1.003-6.275)$ & 0.032 \\
\hline $\begin{array}{l}\text { Age } \\
\leq 60 \text { vs }>60\end{array}$ & $0.778(0.337-1.799)$ & 0.557 & & \\
\hline $\begin{array}{l}\text { Lymph Node Metastasis } \\
\text { Yes vs no }\end{array}$ & $0.723(0.247-2.122)$ & 0.531 & & \\
\hline $\begin{array}{l}\text { TNM stage } \\
\text { III and IV vs I and II }\end{array}$ & $1.435(0.627-3.285)$ & 0.393 & & \\
\hline $\begin{array}{l}\text { Grade } \\
\text { G3 and } G 4 \text { vs GI and G2 }\end{array}$ & I.504 (0.67I-3.372) & 0.322 & & \\
\hline $\begin{array}{l}\text { HBV Infection } \\
\text { Yes vs no } \\
\text { NASH, yes vs no }\end{array}$ & $\begin{array}{l}2.013(1.537-4.28 I) \\
1.394(1.027-2.116)\end{array}$ & $\begin{array}{l}0.009 \\
0.041\end{array}$ & & \\
\hline $\begin{array}{l}\text { Alcohol abuse } \\
\text { Yes vs no }\end{array}$ & $0.907(0.213-1.408)$ & 0.059 & & \\
\hline $\begin{array}{l}\text { Recurrence } \\
\text { Yes vs no }\end{array}$ & $2.707(1.910-4.552)$ & 0.011 & & \\
\hline
\end{tabular}

had received treatment, nevertheless, quite a few patients still suffered from tumor recurrence (distant or locoregional) even after hepatic resection. ${ }^{14}$ The initiation and progression of HCC was regulated by various genetic changes. Thus, identifying the dysregulated genes in tumor progression could be helpful for improving prognostic and therapeutic strategies. PZP, as a pregnancyassociated protein, is dramatically upregulated in the maternal plasma and plays an important role during gestation. Cater et al found that PZP could efficiently inhibit the aggregation of misfolded proteins including amyloid beta peptide and maintain protein homeostasis during pregnancy, indicating that PZP might play a potential role in the development of preeclampsia and Alzheimer's disease. ${ }^{4}$ Furthermore, as a powerful $\mathrm{T}$ cell immunosuppressant for preventing rejection of the fetal allograft during pregnancy, PZP was associated with airway infection, disease severity in bronchiectasis during chronic airway inflammation. ${ }^{15}$ However, in the previous studies, the relationship between PZP expression and tumor, especially HCC was not reported.

In our present study, bioinformatics analysis was first performed to explore the role of PZP expression in HCC.
We found that PZP expression was decreased in HCC tissues than that in normal tissues, and the decreased PZP expression was positively correlated with worse prognosis in patients with $\mathrm{HCC}$, according to the results analyzed by GEPIA and HPA. At the same time, TCGA database for LIHC was downloaded and analyzed, the results of which revealed that PZP expression was an independent prognostic factor.

Second, with the aim of verifying the results of bioinformatics analysis, we collected $59 \mathrm{HCC}$ samples and 30 corresponding noncancerous tissues to perform IHC and qPCR assay, meanwhile, Kaplan-Meier survival analysis and Cox proportional regression analysis were carried out. Fortunately, all of the results of above were in accord with the results of bioinformatics analysis.

Third, we explored the relationship between immune infiltration levels and PZP expression in HCC by TIMER and CIBERSORT, and found that PZP expression had a positive correlation with the levels of resting memory CD4 T cells and Tregs in HCC immune microenvironment. In order to confirm the association between PZP expression and TIICs in HCC microenvironment, IHC assay of CD4+ T cells and Treg cells in HCC tissues was 

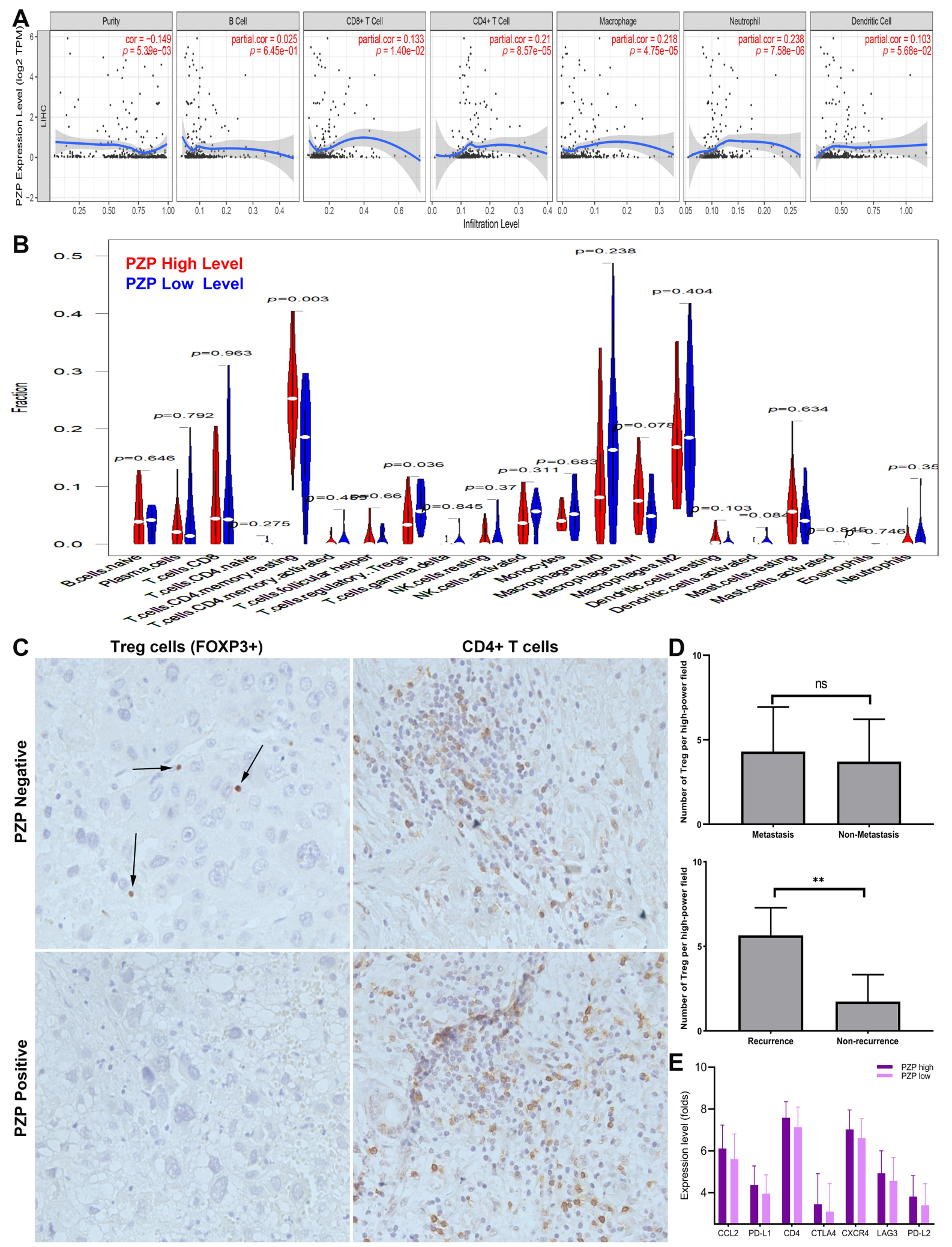

Figure 3 Association of PZP expression and TIICs in HCC microenvironment. Associations between PZP expression and immune infiltration levels in HCC analyzed by TIMER (A) and CIBERSORT (B). The IHC assay results of CD4+ T cells and Treg cells in HCC tissues (C). The difference of Treg cells between patients with or without metastasis or recurrence (D). High PZP group exhibited higher expression of seven immune checkpoint genes $(\mathbf{E})$. ${ }^{*} p<0.01$, $* * p>0.05$. 
performed, the results of which showed that only Treg was negatively associated with PZP expression.

Forth and finally, we investigated the association between PZP and 15 potentially targetable immune checkpoint genes in TCGA database, the results of which showed that high PZP group exhibited higher expression of seven immune checkpoint genes, including CCL2, PDL1, CD4, CTLA4, CXCR4, LAG3 and PDL2, than low PZP group. All these above indicated that PZP expression had a significant meaning in the progression and in regulating tumor immune microenvironment of $\mathrm{HCC}$.

A limitation of the present study was that the PZP expression levels were only detected in tissues by IHC and qPCR assay, analysis in vivo and vitro are required to support our results. Further, our study is a cohort with a small sample size. Data from future well-designed studies involving multiple centers and large numbers of patients will be required to provide rigorous assessment and validation of the efficacy of our conclusions.

In conclusion, our study is the first analysis to demonstrate that the expression of PZP was decreased in HCC tissues, and the PZP-low population has a positively association with poor prognosis in patients with HCC. Moreover, PZP might play a vital role in the regulation and recruitment of TIICs in HCC immune microenvironment. In addition, the results of the present study confirmed that PZP expression might be a prognostic biomarker and potential molecular therapeutic target for HCC.

\section{Ethics Approval and Consent to Participate}

The present study was approved by the Ethics Committees of Zhengzhou University. Written informed consent was obtained from all patients.

\section{Acknowledgments}

Lisa $\mathrm{Su}$ and Genhao Zhang are co-first authors for this study.

\section{Funding}

There is no funding to report.

\section{Disclosure}

The authors report no conflicts of interest in this work.

\section{References}

1. Liu Y, Liu L, Zhou Y, et al. CKLF1 enhances inflammation-mediated carcinogenesis and prevents doxorubicin-induced apoptosis via IL6/ STAT3 signaling in HCC. Clin Cancer Res. 2019;25(13):4141-4154. doi:10.1158/1078-0432.CCR-18-3510

2. Shen H, Wang Z, Ren S, et al. Prognostic biomarker MITD1 and its correlation with immune infiltrates in hepatocellular carcinoma (HCC). Int Immunopharmacol. 2020;81:106222. doi:10.1016/j. intimp.2020.106222

3. Fujiwara N, Friedman SL, Goossens N, Hoshida Y. Risk factors and prevention of hepatocellular carcinoma in the era of precision medicine. J Hepatol. 2018;68(3):526-549.

4. Cater JH, Kumita JR, Zeineddine Abdallah R, et al. Human pregnancy zone protein stabilizes misfolded proteins including preeclampsia- and Alzheimer's-associated amyloid beta peptide. Proc Natl Acad Sci U S A. 2019;116(13):6101-6110. doi:10.1073/ pnas. 1817298116

5. Krause K, Azouz F, Nakano E, Nerurkar VR, Kumar M. Deletion of pregnancy zone protein and murinoglobulin-1 restricts the pathogenesis of west nile virus infection in mice. Front Microbiol. 2019;10:259. doi:10.3389/fmicb.2019.00259

6. Skornicka EL, Kiyatkina N, Weber MC, Tykocinski ML, Koo PH. Pregnancy zone protein is a carrier and modulator of placental protein-14 in T-cell growth and cytokine production. Cell Immunol. 2004;232(1-2):144-156. doi:10.1016/j.cellimm.2005.03.007

7. Tang Z, Li C, Kang B, Gao G, Li C, Zhang Z. GEPIA: a web server for cancer and normal gene expression profiling and interactive analyses. Nucleic Acids Res. 2017;45(W1):W98-w102. doi:10.1093/ nar/gkx247

8. Zhang G. Expression and prognostic significance of BANF1 in triple-negative breast cancer. Cancer Manag Res. 2020;12:145-150. doi:10.2147/CMAR.S229022

9. Livak KJ, Schmittgen TD. Analysis of relative gene expression data using real-time quantitative PCR and the 2(-Delta Delta C(T)) method. Methods. 2001;25(4):402-408. doi:10.1006/meth.2001.1262

10. Li T, Fan J, Wang B, et al. TIMER: a web server for comprehensive analysis of tumor-infiltrating immune cells. Cancer Res. 2017;77(21): e108-e110. doi:10.1158/0008-5472.CAN-17-0307

11. Gentles AJ, Newman AM, Liu CL, et al. The prognostic landscape of genes and infiltrating immune cells across human cancers. Nat Med. 2015;21(8):938-945. doi:10.1038/nm.3909

12. Yang C, Huang X, Liu Z, Qin W, Wang C. Metabolism-associated molecular classification of hepatocellular carcinoma. Mol Oncol. 2020;14(4):896-913. doi:10.1002/1878-0261.12639

13. Fu H, Zhu Y, Wang Y, et al. Identification and validation of stromal immunotype predict survival and benefit from adjuvant chemotherapy in patients with muscle-invasive bladder cancer. Clin Cancer Res. 2018;24(13):3069-3078. doi:10.1158/1078-0432.CCR-17-2687

14. Li Z, Gao J, Zheng SM, et al. The efficacy of sorafenib in preventing hepatocellular carcinoma recurrence after resection: a systematic review and meta-analysis. Rev Esp Enferm Dig. 2020;112. doi:10.17235/reed.2020.6458/2019

15. Finch S, Shoemark A, Dicker AJ, et al. Pregnancy zone protein is associated with airway infection, neutrophil extracellular trap formation, and disease severity in bronchiectasis. Am J Respir Crit Care Med. 2019;200(8):992-1001. doi:10.1164/rccm.201812-2351OC 


\section{Publish your work in this journal}

Cancer Management and Research is an international, peer-reviewed open access journal focusing on cancer research and the optimal use of preventative and integrated treatment interventions to achieve improved outcomes, enhanced survival and quality of life for the cancer patient.
The manuscript management system is completely online and includes a very quick and fair peer-review system, which is all easy to use. Visit http://www.dovepress.com/testimonials.php to read real quotes from published authors.

Submit your manuscript here: https://www.dovepress.com/cancer-management-and-research-journal 